Bull. Mater. Sci., Vol. 7, No. 5, December 1985, pp. 483-489. (C) Printed in India.

\title{
Non-ohmic electric conduction in polycrystalline thulium oxide
}

\author{
N C PARAKH† and J C GARG* \\ Department of Physics, University of Rajasthan, Jaipur 302004, India \\ †On leave from MLv Government College, Bhilwara 311001 , India \\ MS received 27 February 1984; revised 27 May 1985
}

\begin{abstract}
The current voltage behaviour of polycrystalline $\operatorname{Tm}_{2} \mathrm{O}_{3}$ has been investigated at $77 \mathrm{~K}$ and $300 \mathrm{~K}$ to $350 \mathrm{~K}$. At low voltages, the conduction is ohmic while at higher voltages the current has a quadratic dependence on voltage. The voltage $V_{T}$ at which the transition from ohmic to non-ohmic conduction takes place changes from $180 \mathrm{~V}$ to $100 \mathrm{~V}$ as temperature increases from $77 \mathrm{~K}$ to $350 \mathrm{~K}$. The results are interpreted in terms of the Rose theory of space charge limited currents (SCLC) in defect insulators containing shallow traps.
\end{abstract}

Keywords. Non-ohmic conduction; space charge limited currents; thulium oxide; electrical conductivity.

\section{Introduction}

Injection current is a powerful probe for the study of transport properties and defect states in insulators. Recently theoretical studies in space charge limited currents (SCLC) has been reported by Gould (1982) and Sharma (1982). Non-ohmic conduction and SCLC have been experimentally studied in GeSeGa (Mycielski et al 1982), SnO (Agarwal and Saxena 1982), $\mathrm{SiO}_{x}$ (Herdt and Adachi 1981), Ge (Koren and Borisenko 1981), $\mathrm{In}_{2} \mathrm{O}_{3}$ (Jain and Garg 1980), $\mathrm{TeO}_{2}$ (Jain and Garg 1979), $\mathrm{Bi}_{2} \mathrm{O}_{3}$ (Arya and Singh 1979; Alvin and Sathianand 1974), $\mathrm{Tm}_{2} \mathrm{O}_{3}$ (Zdanowicz and Zdanowicz 1979; Zdanowicz et al 1980), $\mathrm{Eu}_{2} \mathrm{O}_{3}$ (Nakane et al 1979), $\mathrm{Dy}_{2} \mathrm{O}_{3}$ (Goswami and Verma 1974) and $\mathrm{TiO}_{2}$ (Katsuta et al 1971). Electric and dielectric properties of rare earth oxide, used in the preparation of garnets, orthoferrites and molybdates have been studied extensively (Brueil et al 1973; Samsonov et al 1974; Lal and Dar 1976; Koleshko and Bapushkina 1979). Electric and dielectric properties of $\mathrm{Tm}_{2} \mathrm{O}_{3}$ (bandgap $5 \cdot 1 \mathrm{eV}$ ) in thin film and pressed pellet form have also been studied (Zdanowicz et al 1980; Lal and Ashok Kumar 1975). Since little information is available in literature on the SCLC in $\mathrm{Tm}_{2} \mathrm{O}_{3}$ we have studied the field dependent electrical conductivity in the $\mathrm{Ag}-\mathrm{Tm}_{2} \mathrm{O}_{3}$ $\mathrm{Ag}$ system as a function of thickness and temperature and the possible nature of defects has been discussed.

\section{Experimental}

Polycrystalline thulium oxide specimens in pellet form have been prepared from specpure (JM grade) powder by pressing the powder at a pressure of $(1-4 \cdot 6) \times 10^{7} \mathrm{kgm} / \mathrm{m}^{2}$

\footnotetext{
* To whom all correspondence should be addressed.
} 
and baking them in an $\mathrm{N}_{2}$ atmosphere at $\sim 1500 \mathrm{~K}$ for $12 \mathrm{hrs}$. After lapping off the surface layers and polishing of the pellets (cross-section area $2.3 \times 10^{-4} \mathrm{~m}^{2}$ ) silver electrodes were deposited by vacuum evaporation on all the samples using molybdenum as boat material at a residual pressure of $5 \times 10^{-5}$ Torr to form $\mathrm{Ag}-\mathrm{Tm}_{2} \mathrm{O}_{3}-\mathrm{Ag}$ sandwich systems. The impurity contents in the starting material are listed below:

$\begin{array}{cccccccc}\text { Element } & \mathrm{Er} & \mathrm{Al} & \mathrm{Ca} & \mathrm{Cu} & \mathrm{Fe} & \mathrm{Ni} & \mathrm{Ta} \\ \text { (ppm) } & 100 & 100 & 1000 & 100 & 200 & 500 & 6000\end{array}$

All the measurements have been carried out in air. The current was measured by an electrometer amplifier (BARC make EA810A). Voltages upto $250 \mathrm{~V}$ were supplied from an electronically regulated power supply of range $0-250 \mathrm{~V}$ and higher voltages $(>250 \mathrm{~V})$ were applied by a regulated power supply of range 250-3000 V (Aplab $7341 \mathrm{P}$ ). The temperature of the specimen was measured by using a precalibrated chromel-alumel thermocouple placed in contact with the specimen. The thermocouple was connected in series with a high impedance panel meter of range $(200 \pm 0.01) \mathrm{mV}$. Sufficient time was allowed for the constancy of the temperature of the specimen while taking observations at different voltages. The observations were repeated and found to be reproducible. The electrical conductivity of the sample at liquid $\mathrm{N}_{2}$ temperature $(77 \mathrm{~K})$ was measured by dipping the sample holder and the connecting leads (properly shielded) directly into a liquid $\mathrm{N}_{2}$ bath. Figures 1a, b show the experimental arrangements for $J-V$ charac-

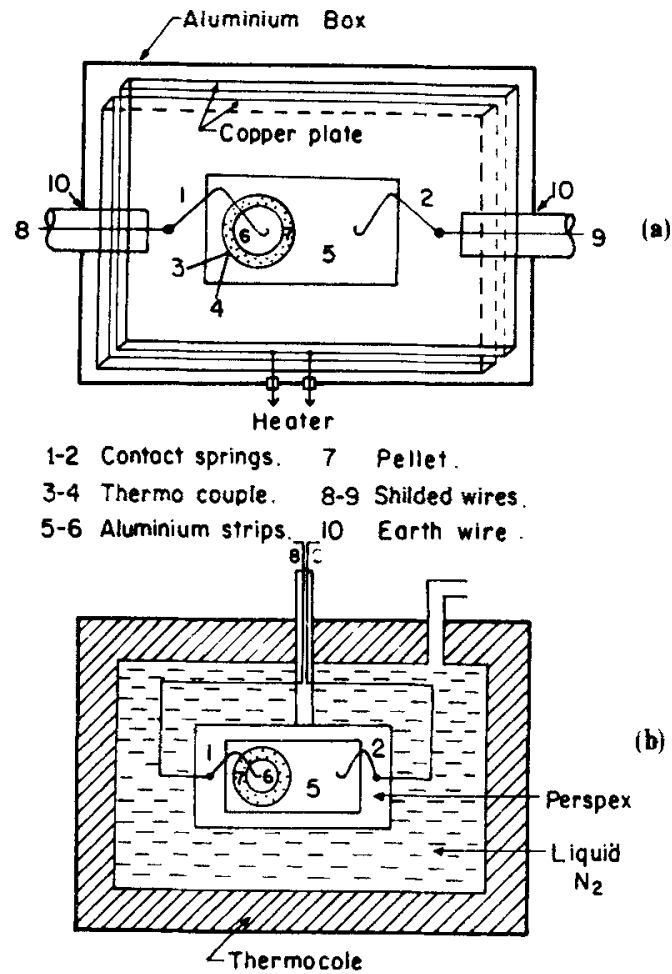

Figure 1. Schematic experimental set-up used for $J-V$ characteristics, (a) at room and high temperatures; (b) at $77 \mathrm{~K}$. 
teristics at room temperature and above and at $77 \mathrm{~K}$ and are self explanatory. Figure 2 shows current density-voltage $(J-V)$ characteristics, on log-log scale for sintered samples prepared at a pressure $1 \times 10^{7} \mathrm{kgm} / \mathrm{m}^{2}$ and having different thicknesses at $300 \mathrm{~K}$. $J$ varies linearly with the applied voltages upto $100-180 \mathrm{~V}$ at different temperatures $(77-350 \mathrm{~K})$ and no change in the magnitude of $J$ is observed while reversing the polarity of the applied voltage. Hence silver contacts formed on the pellets are ohmic. Electrical conductivity is found to vary linearly with the pressure $[(1-4 \cdot 6)$ $\left.\times 10^{7} \mathrm{kgm} / \mathrm{m}^{2}\right]$ applied while preparing the samples. Figure 3 , on the other hand, illustrates the $J-V$ characteristics of one of the samples (thickness $0.8872 \times 10^{-3} \mathrm{~m}$ ) at different temperatures. $J-V$ characteristics follow the same trend in samples prepared at different pressures from $(1-4.6) \times 10^{7} \mathrm{kgm} / \mathrm{m}^{2}$.

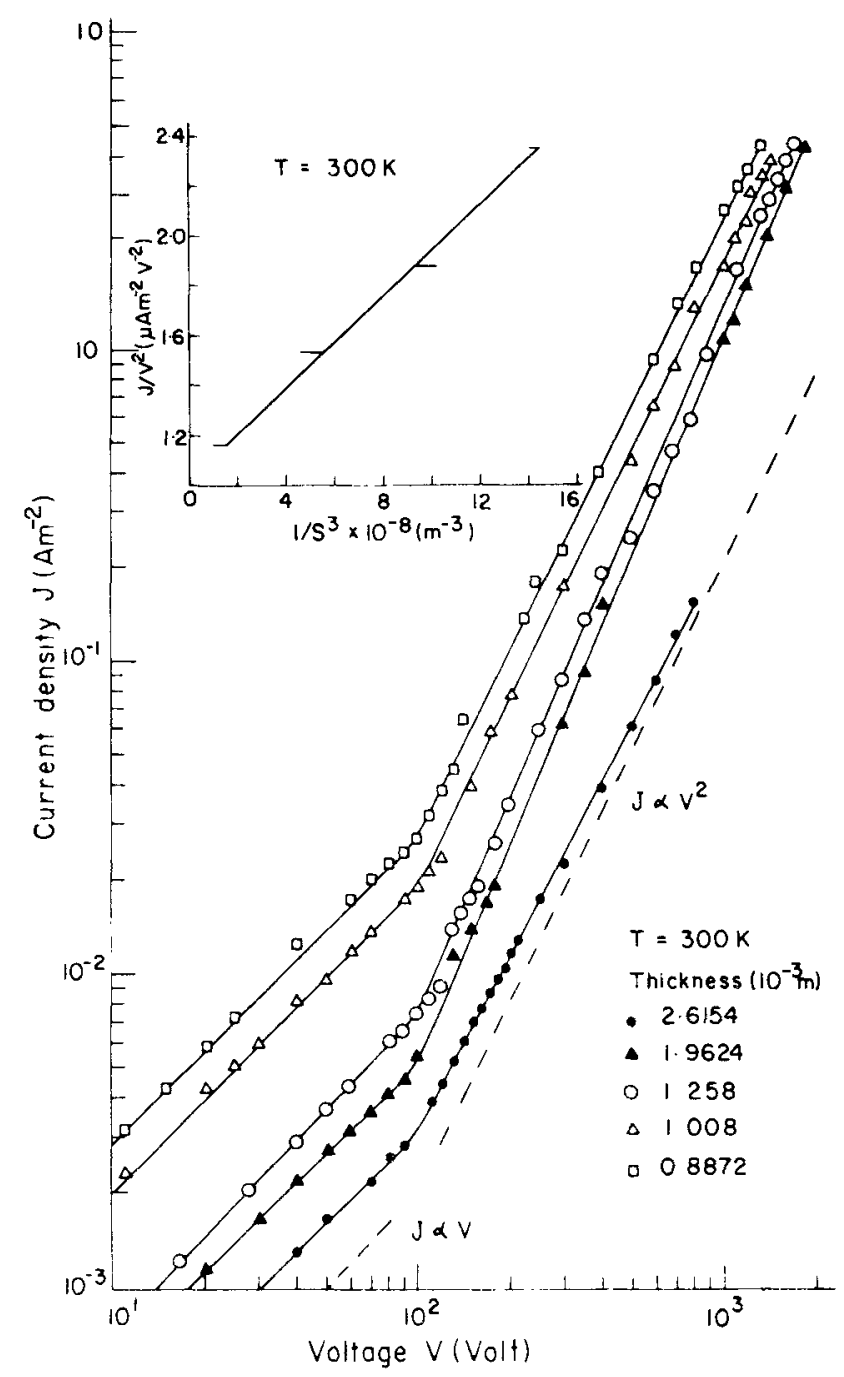

Figure 2. $J-V$ characteristics at $300 \mathrm{~K}$ for pellets of different thicknesses -2.6154 $\times 10^{-3} \mathrm{~m}, \Delta 1.9624 \times 10^{-3} \mathrm{~m}, 01.258 \times 10^{-3} \mathrm{~m}, \Delta 1.008 \times 10^{-3} \mathrm{~m}, \square 0.8872 \times 10^{-3} \mathrm{~m}$. (Inset) Variation of $J / V^{2}$ with $s^{-3}$ at $300 \mathrm{~K}$. 


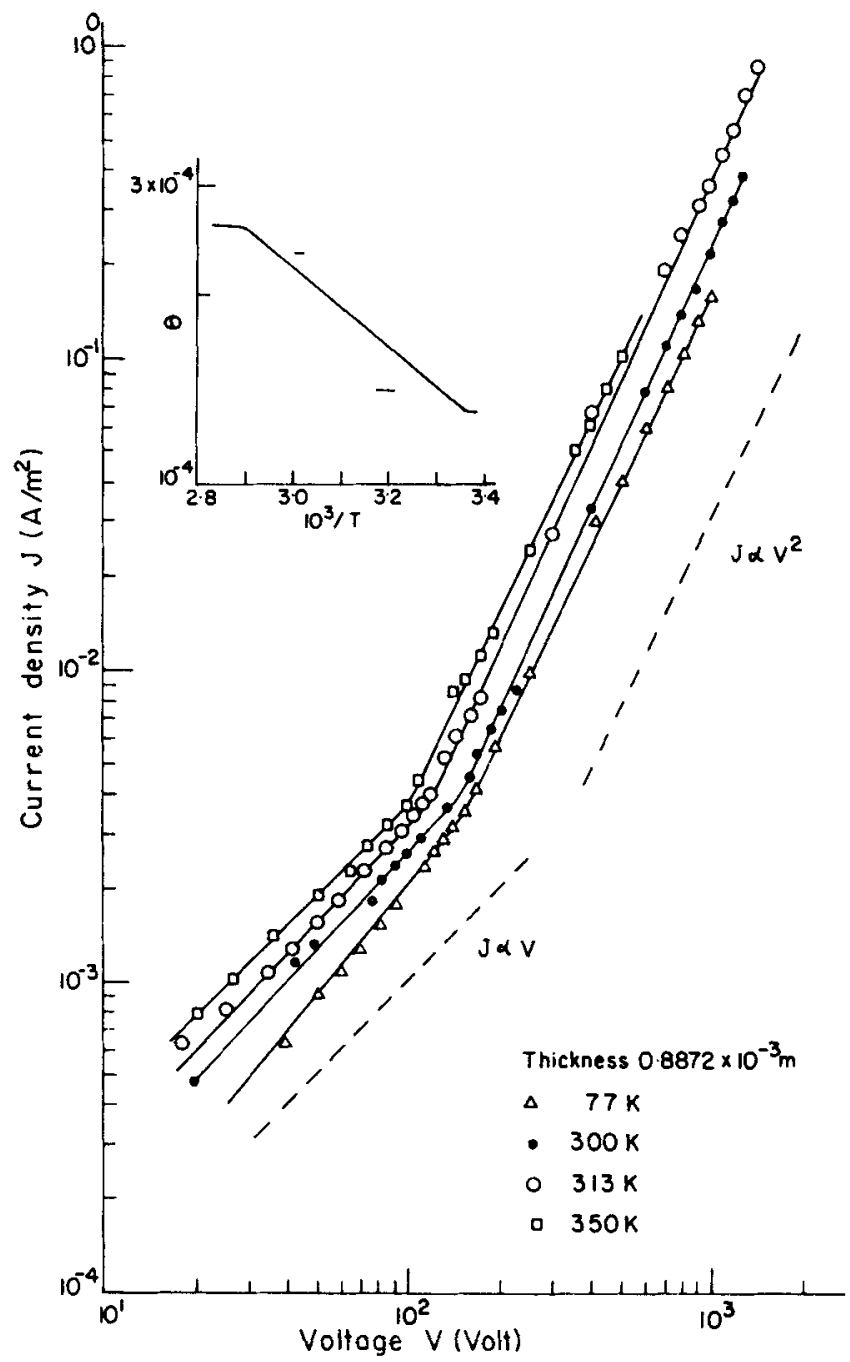

Figure 3. $J-V$ characteristics for a pellet $\left(s=0-8872 \times 10^{-3} \mathrm{~m}\right)$ at different temperatures $\Delta 77 \mathrm{~K}, \bullet 300 \mathrm{~K}, 0313 \mathrm{~K}, \square 350 \mathrm{~K}$. (Inset) Variation of $\ln \theta$ with $10^{3} / T$.

\section{Discussions}

$J-V$ characteristics (figures 2 and 3) show that in all samples at low voltages, the conduction is ohmic while at higher voltages, the current has a quadratic dependence on voltages. It is also seen that transition voltage $\left(V_{T}\right)$ varies from $180 \mathrm{~V}$ to $100 \mathrm{~V}$ as temperature increases from $77 \mathrm{~K}$ to $350 \mathrm{~K}$ (table 1). The observed ohmic dependence of electrical conductivity at low fields can be explained by the fact that in the studied samples bulk limited current exceeds the scLc. The plots of $\log J / V^{2}$ versus $V^{-1}$ are found to be nonlinear (not shown) which means that non-ohmic regions in $J-V$ curves can not be explained by the tunnel effect of Fowler-Nordheim type. The plots of $\log J$ versus $V^{1 / 2}$ and $\log \left(J-J_{0}\right)$ which is the algebraic subtraction of ohmic current density $J_{0}$ from the total current density $J$ versus $V^{1 / 2}$ have also been found to be nonlinear 
(not shown), thereby rejecting the possibility of an explanation of $J-V$ curves on the basis of the Rose theory of exponential trap distribution and the Poole-Frenkel effect respectively. Hence the non-ohmic portions of the $J-V$ curves might be explained on the basis of the shallow trap model (Rose 1955), according to which current density $J$ is given by

$$
J=\left(9 \mu \varepsilon_{r} \varepsilon_{0} \theta V^{2}\right) /\left(8 s^{3}\right)
$$

where $\mu$ is the mobility, $\varepsilon_{r}$ the relative permittivity of the medium, $\varepsilon_{0}$ the permittivity of

Table 1. The values of $V_{T}, \theta$ and $n_{0}$ at different temperatures

\begin{tabular}{cccc}
\hline$T(K)$ & $\begin{array}{c}V_{T} \\
(\text { Volts })\end{array}$ & $\theta \times 10^{5}$ & $n_{0} \times 10^{-12}\left(\mathrm{~m}^{-3}\right)$ \\
\hline 350 & 100 & $3.268 \pm 0.049$ & 2.5228 \\
323 & 120 & $2.937 \pm 0.059$ & 2.7207 \\
313 & 135 & $1.753 \pm 0.049$ & 1.8267 \\
300 & 165 & $1.635 \pm 0.009$ & 2.0825 \\
77 & 180 & $1.145 \pm 0.053$ & 1.5910 \\
\hline
\end{tabular}

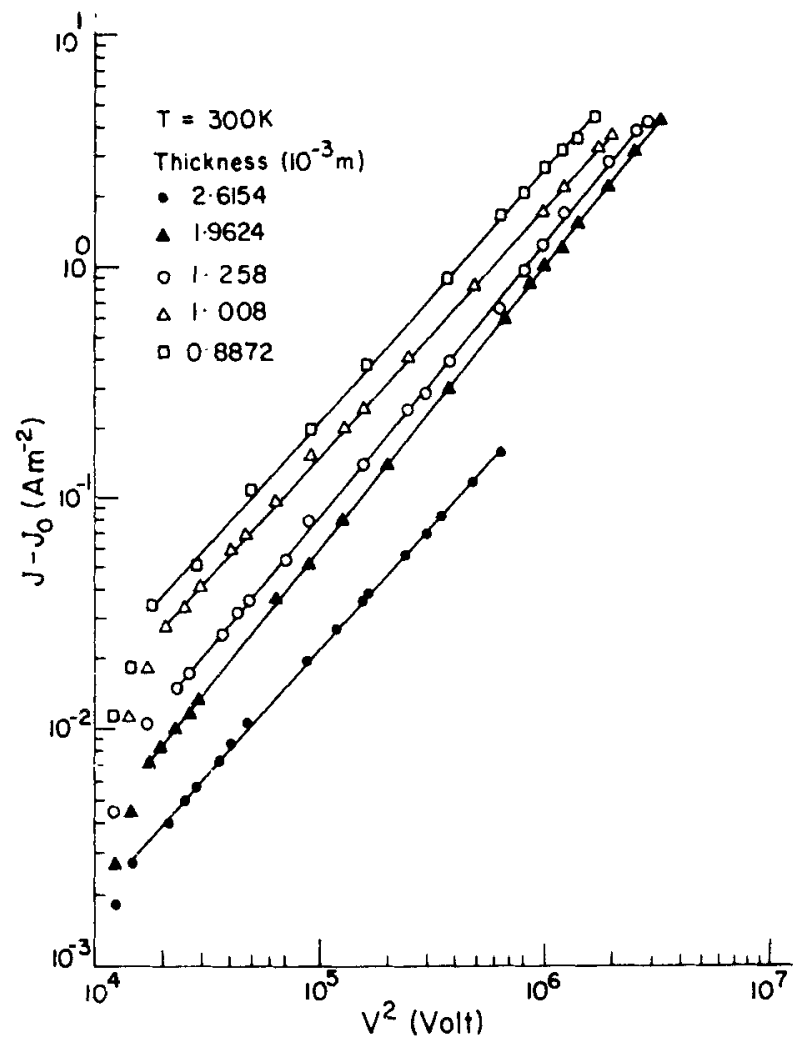

Figure 4. Variation of $J-J_{0}$ with $V^{2}$ at $300 \mathrm{~K}$ for pellets of different thicknesses -2.6154 $\times 10^{-3} \mathrm{~m}, \Delta 1.9624 \times 10^{-3} \mathrm{~m}, 01.258 \times 10^{-3} \mathrm{~m}, \Delta 1.008 \times 10^{-3} \mathrm{~m}, \square 0.8872 \times 10^{-3} \mathrm{~m}$. 
free space, $s$ the thickness of the specimen, $V$ the applied voltage and

$$
\theta=\left(N_{c} / N_{t}\right) \exp \left(-E_{t} / k_{B} T\right)
$$

where $N_{c}$ is the effective density of states in the conduction band, $N_{t}$ is the trap density of a shallow trap at energy $E_{t}$ below the conduction band and $k_{B}$ the Boltzmann constant; it was assumed that free space charge density is negligible in comparison to the total trapped space charge density $(\theta \ll 1)$. Voltage at which conduction changes from ohmic to non-ohmic is given by (Lampert et al 1959):

$$
V_{T}=\left(e n_{0} s^{2}\right) /\left(\theta \varepsilon_{r} \varepsilon_{0}\right)
$$

where $e$ is electronic charge and $n_{0}$ the volume generated free carriers.

The straight lines obtained in $J-J_{0}$ versus $V^{2}$ curves on the $\log$-log scale in the square law region for pellets of different thickness at $300 \mathrm{~K}$ (figure 4) and for a pellet of thickness $0.8872 \times 10^{-3} \mathrm{~m}$ at different temperatures (figure 5) show the validity of (1). The straight line (inset of figure 2) in $J / V^{2}$ versus $s^{-3}$ graph further supports the

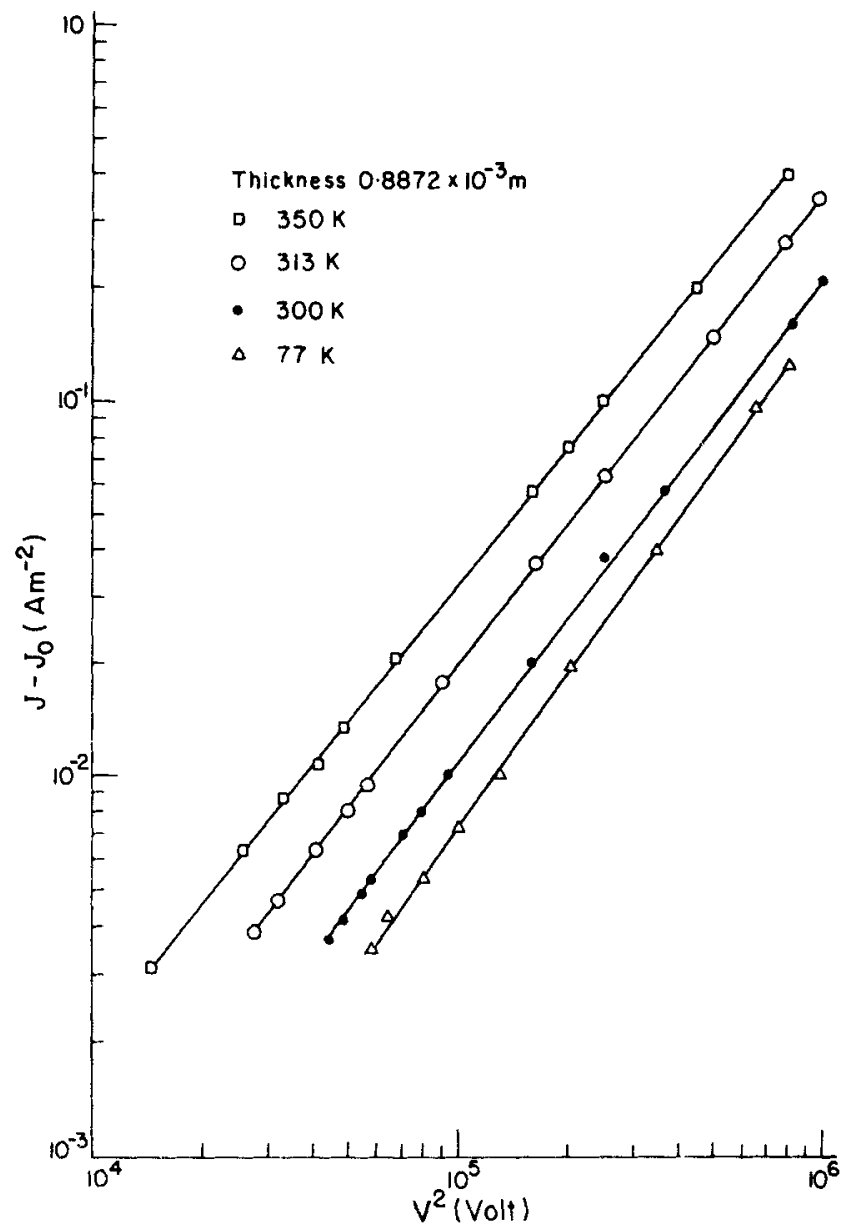

Figure 5. Variation of $J-J_{0}$ with $V^{2}$ for a pellet of thickness $0.8872 \times 10^{-3} \mathrm{~m}$ at different temperatures $-\triangle 77 \mathrm{~K} \odot 300 \mathrm{~K}, \bigcirc 313 \mathrm{~K}, \square 350 \mathrm{~K}$. 
validity of (1). The horizontal bars in the graph represent the errors in the measurement of $s$.

The calculated values of $\theta$ from (1) by the least square fit method taking $\varepsilon_{\mathrm{r}}=11$ (Koleshko and Bapushikna 1979), $\mu=10^{-2} \mathrm{~m}^{2} / V$ sec (assumed) are listed in table 1. It also includes the values of transition voltages $\left(V_{T}\right)$ obtained from figure 3 and $n_{0}$ calculated from (3).

The plot of $\ln \theta$ versus $10^{3} / T$ has been represented in the inset of figure 3 where horizontal bars represent the errors in the measurement of temperature. Assuming that $N_{c}$ and $N_{t}$ are not strong functions of temperatures, the intercept at $10^{3} / T$ being equal to zero yields as ratio $N_{c} / N_{t}=1.8 \times 10^{-2}$ which on substitution in (2) gives a trap level at a depth of $\sim 0.12 \mathrm{eV}$ below the conduction band.

\section{Conclusions}

The quadratic dependence of current density on voltage $\left(J \propto V^{2}\right)$ in the non-ohmic region of $J-V$ characteristics in polycrystalline $\mathrm{Tm}_{2} \mathrm{O}_{3}$ at different temperatures has been interpreted in terms of SCLC in defect insulators containing shallow traps which exist at energy level $\sim 0 \cdot 12 \mathrm{eV}$ below the conduction band.

\section{Acknowledgement}

One of the authors (NCP) is grateful to the UGC, New Delhi for the award of a fellowship. The authors are thankful to Prof. S Lokanathan for encouragement.

\section{References}

Agarwal T N and Saxena R N 1982 Indian J. Pure Appl. Phys. 20780

Alvin S and Sathianand K 1974 Thin Solid Films 24539

Arya S P S and Singh H P 1979 Thin Solid Films 62353

Brueil H, Dherbomez N and Wilbert Y 1973 C. R. Hebd. Seances Acad. Sci. C277 871

Goswami A and Verma R R 1974 Thin Solid Films 2252

Gould R D 1982 J. Appl. Phys. 533753

Herdt $W$ and Adachi $\mathbf{H} 1981$ Thin Solid Films 82293

Jain D K and Garg J C 1979 Indian J. Pure Appl. Phys. 17642

Jain D K and Garg J C 1980 Indian J. Pure Appl. Phys. 18842

Katsuta Y, Akhane R and Yahagi K 1971 Jpn. J. Appl. Phys. 10976

Koleshko V M and Bapushkina N V 1979 Thin Solid Films 621

Koren N N and Borisenko T F 1981 Phy.. Status Solidi K67 147

Lal H B and Ashok Kumar 1975 Proc. Nucl. Phys. Solid State Phys. Symp. C18 121

Lal H B and Dar N 1976 Indian J. Pure Appl. Phy's. 14788

Lampert M A. Rose A and Smith R W 1959 J. Phys. Chem. Solids 8464

Mycielski W. Lach A and Lipinski A 1982 Thin Solid Films 84 L177

Nakane H, Noya A. Kuriki S and Matsumoto G 1979 Thin Solid Films 84 L177

Rose A 1955 Phys. Rev. 971538

Samsonov G V, Gilman Iya and Andreeva A F 1974 Izl. Akad. Nauk SSSR, Neorg. Mater. 101645

Sharma Y K 1982 J. Appl. Phys. 533753

Zdanowicz T and Zdanowicz L 1979 Thin Solid Films $\mathbf{5 8} 390$

Zdanowicz T, Jankowski B and Zdanowicz L 1980 Acta Phys. Pol. A57 151 